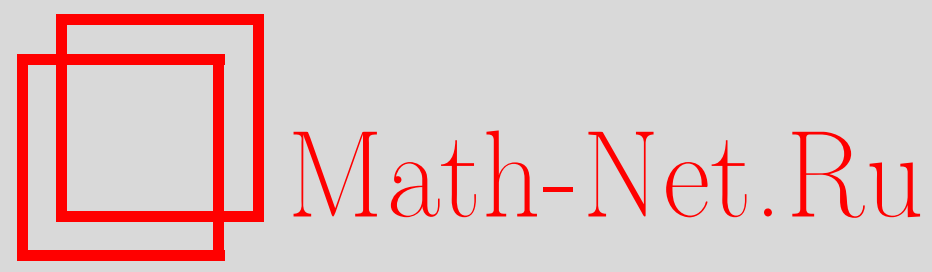

Н. Т. Лупашко, Об автоморфизмах коммутативных луп Муфанг, Дискрет. матем., 2011, том 23, выпуск 2, 108-114

DOI: https://doi.org/10.4213/dm1146

Использование Общероссийского математического портала Math-Net.Ru подразумевает, что вы прочитали и согласны с пользовательским соглашением http://www.mathnet.ru/rus/agreement

Параметры загрузки:

IP: 54.147 .182 .235

26 апреля 2023 г., 12:13:41 


\title{
Об автоморфизмах коммутативных луп Муфанг
}

\author{
() 2011 г. $\quad$ Н. Т. Лупашко
}

\begin{abstract}
Доказывается, что группа автоморфизмов произвольной коммутативной лупы Муфанг $Q$ является расширением группы $F(1)$, состоящая из всех автоморфизмов лупы $Q$, которые индуцируют тождественное отображение фактор-лупs $Q$ по ее ассоциаторной подлупе $A(Q)$, с помощью группы автоморфизмов абелевой группы $Q / A(Q)$. Исследуется строение группы $F(1)$ в случаях, когда лупа $Q$ центрально нильпотентна, или конечно порождена, или является $Z A$-лупой.
\end{abstract}

Одним из наиболее изученным классом неассоциативных луп является класс коммутативных луп Муфанг. В настоящей работе изучается строение группы автоморфизмов $F$ таких луп $Q$. Пусть $A(Q)$ - ассоциаторная подлупа лупы $Q$, и пусть $F(1)-$ множество всех автоморфизмов из $F$, которые индуцируют тождественное отображение на фактор-лупе $Q / A(Q)$. Доказывается, что группа $F$ является расширением группы $F(1)$ с помощью группы автоморфизмов абелевой группы $Q / A(Q)$. Исследуется строение группы $F(1)$ при условии, что лупа $Q$ является центрально нильпотентной или $Z A$-лупой, или конечно порожденной. Отметим, что группы автоморфизмов коммутативных луп Муфанг исследуются в [1], а в [2, 3] исследуются группы внутренних подстановок таких луп, которые являются подгруппами групп автоморфизмов.

Теперь напомним некоторые понятия и результаты из теории луп, которые можно найти в [3].

Коммутативные лупы Муфанг (кратко КЛМ) характеризуются тождеством

$$
x^{2} \cdot y z=x y \cdot x z \text {. }
$$

Группа внутренних подстановок $I(Q)$ КЛМ $Q$ - это группа, порожденная всеми внутренними подстановками $L(u, v)=L(u v)^{-1} L(u) L(v)$, где $u, v \in Q$ и $L(x) y=x y$. Для элементов $a, b, c \in Q$ ассоциатор $(a, b, c)$ определяется равенством

$$
a b \cdot c=(a \cdot b c)(a, b, c) .
$$

Подлупа $A(Q)$, порожденная всеми ассоциаторами КЛМ $Q$, называется ассоциаторной подлупой КЛМ $Q$. Центр КЛМ $Q$ - это нормальная подлупа

$$
Z(Q)=\{x \in Q \mid(x, y, z)=1 \forall y, z \in Q\} .
$$

В произвольной КЛМ справедливы тождества

$$
\begin{aligned}
L(x, y) z & =z(z, y, x), \\
(x, y, z) & =\left(y^{-1}, x, z\right)=(y, x, z)^{-1}=(y, z, x), \\
(x, y, z)^{3} & =1, \\
(x y, u, v) & =(x, u, v)((x, u, v), x, y) \cdot(y, u, v)((y, u, v), y, x) .
\end{aligned}
$$


Возрастающим центральным рядом некоторой КЛМ $Q$ называется вполне упорядоченная по включению последовательность

$$
Z_{0}=1 \subset Z_{1} \subset Z_{2} \subset \ldots \subset Z_{\alpha} \subset \ldots \subset Z_{\gamma}=Q
$$

нормальных подлуп КЛМ $Q$, удовлетворяющая следующим условиям:

(1) $Z_{\alpha}=\sum_{\beta<\alpha} Z_{\beta}$ при предельном порядковом числе $\alpha$, и

(2) фактор-лупа $Z_{\alpha+1} / Z_{\alpha}$ - фактор ряда (5) при любом $\alpha$ содержится в центре факторлупы $Q / Z_{\alpha}$.

Возрастающий центральный ряд (5) КЛМ $Q$ называется ее верхним центральным рядом, если его фактор $Z_{\alpha+1} / Z_{\alpha}$ при любом $\alpha$ совпадает с центром фактор-лупы $Q / Z_{\alpha}$. КЛМ, обладающая возрастающим центральным рядом, называется $Z A$-лупой. Если верхний центральный ряд $Z A$-лупы конечен, то она называется центрально нильпотентной, а число факторов такого ряда - ступенью центральной нильпотентности.

Для КЛМ $Q$ обозначим $A_{1}(Q)=A(Q)$ и по индукции определяем $A_{n+1}(Q)$ как подлупу, порожденную всеми ассоциаторами $(u, x, y)$, где $u \in A_{n}(Q), x, y \in Q$. Получим ряд нормальных подлуп

$$
Q=A_{0}(Q) \supseteq A_{1}(Q) \supseteq A_{2}(Q) \supseteq \ldots \supseteq A_{i}(Q) \supseteq \ldots,
$$

который называется нижним центральным рядом КЛМ $Q$. КЛМ $Q$ центрально нильпотентна ступени $n$, если ее верхний и нижний центральные ряды соответственно имеют вид

$$
1 \subset Z_{1} \subset \ldots \subset Z_{n}=Q, \quad Q \supset A_{1}(Q) \supset \ldots \supset A_{n}(Q)=1 .
$$

Наиболее глубоким результатом теории КЛМ является теорема Брака-Слэби: КЛМ с $n$, $n>1$, порождающими центрально нильпотентна ступени не более чем $n-1$.

В $[4,5,6]$ построены примеры КЛМ с $n$ порождающими, которые являются центрально нильпотентными ступени $n-1$. Поэтому верно следующее утверждение.

Лемма 1. Свободная КЛМ с п свободными порождающими цุентрально нильпотентна ступени $n-1$.

Пусть теперь $Q-$ произвольная КЛМ с нижним центральным рядом (6). Через $F$ обозначим группу автоморфизмов КЛМ $Q$, а через $F(k), k=1,2, \ldots,-$ множество всех автоморфизмов из $F$, которые индуцируют тождественное отображение на $Q / A_{k}(Q)$. Легко показать, что $F(k)$ является нормальной подгруппой группы $F$.

Лемма 2 ([1]). Для $m=1,2, \ldots$ и для каждого неотрицательного числа $k$ элементьл группь $F(k)$ индуцииуют тождественное отображение на $A_{m}(Q) / A_{m+k}(Q)$.

Через $F(1)=F_{0} \supseteq F_{1} \supseteq \ldots \supseteq F_{i} \supseteq \ldots$ обозначим нижний центральный ряд подгруппы $F(1)$ группы $F$. Справедливо следующее утверждение.

Лемма 3. Для любого ичелого неотрицательного $i$ справедливо включение $F_{i} \subseteq F(i+1)$. Если $\varphi \in F(i)$, то $\varphi^{3} \in F(2 i)$. Более того, $\varphi^{f(i)} \in F(g(i))$ для $\varphi \in F(i)$, где $f(i)=3^{i}$, $g(i)=2^{i}$. 
Доказательство. Справедливо равенство $F_{0}=F(1)$. Предположим, что $F_{i-1} \subseteq F(i)$. В [1] доказано, что для любых целых неотрицательных чисел $k, m$ верно соотношение $(F(m), F(k)) \subseteq F(m+k)$, где $(F(m), F(k))$ означает подгруппу, порожденную всеми коммутаторами $(u, v), u \in F(m), v \in F(k)$. Тогда

$$
F_{i}=\left(F_{i-1}, F_{0}\right)=\left(F_{i-1}, F(1)\right) \subseteq(F(i), F(1)) \subseteq F(i+1) .
$$

Поэтому $F_{i} \subseteq F(i+1)$ для любого целого числа $i$.

Пусть теперь $\varphi \in F(i), x \in Q$. Согласно определению подгруппы $F(i)$, справедливо равенство $\varphi x=x y$, где $y \in A_{i}(Q)$. По лемме 2,

$$
\varphi y=y \quad(\bmod A)_{2 i}(Q) .
$$

КЛМ диассоциативна, поэтому

$$
\varphi^{2} x=\varphi(x y)=\varphi x \cdot \varphi y=(x y \cdot y) \quad(\bmod A)_{2 i}(Q)=\left(x y^{2}\right) \quad \bmod A_{2 i}(Q) .
$$

Аналогично,

$$
\varphi^{3} x=\left(x y^{3}\right) \quad(\bmod A)_{2 i}(Q) .
$$

Но $y \in A_{i}(Q)$ и $i>0$, поэтому, согласно (3), $y^{3}=1$. Отсюда следует, что $\varphi^{3} \in F(2 i)$.

Покажем, что из $\varphi \in F(1)$ следует включение $\varphi^{f(i)} \in F(g(i))$ для любого целого числа $i \geqslant 1$. Действительно, для $i=1$ утверждение доказано выше. Пусть $\varphi^{f(i-1)} \in F(g(i-1))$. Тогда

$$
\varphi^{f(i)}=\left(\varphi^{f(i-1)}\right)^{3} \in F(g(i-1) \cdot 2)=F(g(i)) .
$$

Лемма доказана.

Следствие 1 ([1]). Пусть $Q$ - иентрально нильпотентная ступени $n$ КЛМ и пусть $F(1)$ - подгруппа группь ее автоморфизмов, определенная выше. Тогда $F(1)$ будет нильпотентной группой ступени не больше $n-1$ и показателя, делящего $3^{r}$, где $r=\min \left\{r \mid 2^{r} \geqslant n\right\}$, и следовательно, будет локально конечной группой.

Следствие 2. Если $Q$ - конечно порожденная КЛМ, то ее подгруппа $F(1)$ группь Aut $Q$ является конечной 3-группой.

Доказательство. Согласно [3], ассоциаторная подлупа произвольной конечно порожденной КЛМ конечна. Пусть КЛМ $Q$ порождается конечным множеством $X$. Из определения группы $F(1)$ следует, что если $\alpha \in F(1)$, то $\alpha$ индуцирует тождественное отображение на $Q / A(Q)$. Поэтому $\alpha x=x h$ для $x \in X$, где $h \in A(Q)$. Очевидно, что для $\alpha, \beta \in F(1)$, $\alpha \neq \beta$, если в записи $\alpha x_{1}=x_{1} h_{1}, \beta x_{2}=x_{2} h_{2}$ хотя бы одна из пар $x_{1}, x_{2}$ или $h_{1}, h_{2}$ содержит различные элементы. Множества $X, A(Q)$ конечны. Тогда число случаев, когда пары $x_{1}, x_{2}$ и $h_{1}, h_{2}$ содержат различные элементы, конечно. Отсюда следует, что группа $F(1)$ конечна. По лемме 1 , КЛМ $Q$ центрально нильпотентна. Тогда из следствия 1 следует, что $F(1)$ является конечной 3-группой. Следствие доказано.

Скажем, что лупа $Q$ аппроксимируется лупами со свойством $\alpha$, если для любого элемента $a$ из $Q$ существует нормальная подлупа $H$ лупы $Q$ такая, что $a \notin H$ и $Q / H$ обладает свойством $\alpha$.

Центрально нильпотентно аппроксимируемые КЛМ - это в точности те КЛМ, у которых равен единице член нижнего центрального ряда с первым бесконечным номером. Такие КЛМ характеризуются поддекартовыми произведениями центрально нильпотентных 
луп. Легко убедиться, используя лемму 1, что свободные КЛМ центрально нильпотентно аппроксимируемы. В [7] приводится пример КЛМ, длина нижнего центрального ряда которой равна произвольному наперед заданному порядковому числу. Отсюда следует, что класс центрально нильпотентно аппроксимируемых КЛМ не замкнут относительно эпиморфных образов.

Лемма 4 ([1]). Если КЛМ $Q$ аппроксимируется иентрально нильпотентныьми КЛМ, то подгруппа $F(1)$ группь автоморфизмов $F$ аппроксимируется нильпотентными группами.

Пусть $L-$ произвольная КЛМ с группой автоморфизмов $F$. Через $J(L)$ обозначим множество всех автоморфизмов из $F$, действующих тождественно на $L / A(L)$, то есть $J(L)=F(1)$. Справедливо следующее утверждение.

Лемма 5. Пусть $Q-$ КЛМ с группой автоморфизмов $F$, пусть $J=F(1)-$ подгруппа группь $F$, и пусть $Z_{i}(Q)=Z_{i}, Z_{i}(J)$ - члены верхних ичентральных рядов лупь $Q u$ группь $J$ соответственно. Тогда

$$
J\left(Q / Z_{i}\right) \cong J / Z_{i}(J)
$$

Доказательство. Справедливо соотношение

$$
J(Q / Z) \cong J / R
$$

где $Z=Z_{1}$,

$$
R=\{\varphi \in J \mid \varphi x \cdot Z=x Z \forall x \in Q\} .
$$

Пусть $\varphi \in R, x, u, v \in Q$. Тогда $\varphi u=u z_{1}, \varphi v=v z_{2}$, где $z_{1}, z_{2} \in Z$. В силу (1) и (4),

$$
\begin{aligned}
\varphi L(u, v) x & =\varphi(x(x, v, u))=\varphi x(\varphi x, \varphi v, \varphi u)=\varphi x\left(\varphi x, v z_{2}, u z_{1}\right)=\varphi x(\varphi x, v, u) \\
& =L(u, v) \varphi x
\end{aligned}
$$

то есть $\varphi L(u, v)=L(u, v) \varphi$ для любых $u, v \in Q$. Это означает, что $\varphi \in Z(J)$, а тогда $R \subseteq Z(J)$.

Обратно, пусть $\varphi \in Z(J)$. Согласно (1) для любых $x, y, z \in Q$

$$
\varphi x(\varphi x, y, z)=L(z, y) \varphi x=\varphi L(z, y) x=\varphi(x(x, y, z))=\varphi x \cdot \varphi(x, y, z),
$$

то есть

$$
\varphi(x, y, z)=\varphi(x, y, z)
$$

Из (8) и (2) получаем, что

$$
\varphi(x, y, z)=(\varphi x, \varphi y, \varphi z)=\varphi^{3}(x, y, z), \quad(x, y, z)=\varphi^{2}(x, y, z) .
$$

Следовательно, автоморфизм $\varphi^{2}$ действует тождественно на $A(Q)$.

Пусть $H-$ произвольная конечно порожденная подлупа КЛМ $Q$ и пусть $\bar{\varphi}-$ сужение $\varphi$ на $A(H)$. Тогда $\bar{\varphi}^{2}$ действует тождественно на $A(H)$. По теореме Брака-Слэби, подлупа $H$ центрально нильпотентна, поэтому по следствию 1 автоморфизм $\bar{\varphi}$ имеет порядок, равный степени числа три. Тогда $\bar{\varphi}$ действует тождественно на $A(H)$. Поскольку $H$ 
является произвольной конечно порожденной подлупой КЛМ $Q$, то тогда и автоморфизм $\varphi$ действует тождественно на $A(Q)$. Следовательно, из (8) следует, что

$$
(\varphi x, y, z)=(x, y, z)
$$

Теперь фиксируем элемент $x$ и определим элемент $a$, полагая $\varphi x=x a$. Из (9) и (4) получаем, что

$$
(x, y, z)=(x a, y, z)=(x, y, z)((x, y, z), x, a) \cdot(a, y, z)((a, y, z) a, x) .
$$

Тогда

$$
(a, y, z)=((x, y, z), x, a)^{-1}((a, y, z), a, x)^{-1}
$$

для любых $y, z \in Q$. Из (10) при $y=x, z=w$ получаем, что $(a, x, w)=1$ для любого $w \in Q$. В частности, оба сомножителя правой части равенства (10) равны единице. Поэтому $a \in Z$, а тогда $\varphi x \cdot Z=x Z$ для любого $x \in Q$, то есть $Z(J) \subseteq R$. Следовательно, $R=Z(J)$.

Предположим, что утверждение леммы верно для некоторого $i$. Тогда из определения верхнего центрального ряда лупы $Q$ и предположения индукции получаем, что

$$
\begin{aligned}
J\left(Q / Z_{i+1}\right) & \cong J\left(\left(Q / Z_{i}\right) /\left(Z_{i+1} / Z_{i}\right)\right)=J\left(\left(Q / Z_{i}\right) / Z\left(Q / Z_{i}\right)\right) \cong J\left(Q / Z_{i}\right) / Z\left(J\left(Q / Z_{i}\right)\right) \\
& \cong\left(J / Z_{i}(J)\right) / Z\left(J / Z_{i}(J)\right) \cong\left(J / Z_{i}(J)\right) /\left(Z_{i+1}(J) / Z_{i}(J)\right) \cong J / Z_{i+1}(J) .
\end{aligned}
$$

Лемма доказана.

Пусть теперь $Q-$ произвольная КЛМ с центром $Z$ и группой внутренних подстановок $I$, и пусть $Z(I)$ - центр группы $I$. В [3] доказано, что $I(Q / Z) \cong I / Z(I)$. Тогда из этого факта, леммы 5 и определений $Z A$-лупы и центрально нильпотентной лупы индукцией мы непосредственно получаем следующие утверждения.

Теорема 1. Для КЛМ $Q$ с группой $F(1)$, состоящеей из автоморфизмов, индуциирующих тождественное отображение на $Q / A(Q)$, и с группой внутренних подстановок $I(Q)$ следующие условия эквивалентны:

(1) КЛМ Q центтрально нильпотентна ступени n;

(2) группа $F(1)$ нильпотентна ступени $n-1$;

(3) группа $I(Q)$ нильпотентна ступени $n-1$.

Предложение 1. Для КЛМ $Q$ с группой $F(1)$, состоящей из автоморфизмов, индуцирующих тождественное отображение на $Q / A(Q)$, и с группой внутренних подстановок $I(Q)$ следующие условия эквивалентны:

(1) $Q$ является ZA-лупой;

(2) $F(1)$ является ZA-группой;

(3) $I(Q)$ является ZA-группой. 
Теперь рассмотрим группы автоморфизмов КЛМ с тривиальным центром, которые, ввиду (7), существенно отличаются от центрально нильпотентных луп. По лемме 1, такие лупы не могут быть конечно порожденными. Пример таких луп можно найти в [3]. Из леммы 5 следует, что если центр КЛМ $Q$ тривиален, то и центр подгруппы $F(1)$ группы автоморфизмов $F$ КЛМ $Q$ также тривиален. В частности, центр группы внутренних подстановок КЛМ $Q$ тоже тривиален. Теперь покажем, что центр $Z(F)$ группы автоморфизмов $F$ КЛМ $Q$ состоит только из автоморфизмов $\varphi$ таких, что $\varphi^{2}=1$. (Очевидно, автоморфизм $x \rightarrow x^{-1}, x \in Q$ принадлежит $\left.Z(F)\right)$. Действительно, пусть $\varphi \in Z(F)$ и пусть $\varphi^{2} \neq 1$. Это значит, что существует элемент $a \in Q$ такой, что $\varphi^{2} a \neq a$. Если $x, y \in Q$, то в силу (8) и (2)

$$
\varphi(a, x, y)=(\varphi a, \varphi x, \varphi y)=\varphi\left(\varphi^{2} a, x, y\right) .
$$

Поэтому $(a, x, y)=\left(\varphi^{2} a, x, y\right)$. Отсюда с использованием (4) и (2) получаем, что $\left(\varphi^{2} a \cdot a^{-1}, x, y\right)=1$ для любых $x, y \in Q$. Это означает, что $1 \neq \varphi^{2} a \cdot a^{-1} \in Z(Q)$. Получили противоречие. Следовательно, центр $Z(F)$ группы автоморфизмов $F$ КЛМ $Q$ состоит только из таких автоморфизмов $\varphi$, что $\varphi^{2}=1$.

Теперь докажем следующие утверждения.

Лемма 6. Эндоморфизм КЛМ $Q$ с условием $A(Q) \neq Q$ является автоморфизмом тогда и только тогда, когда он индуцииует автоморфизм на $Q / A(Q)$.

Доказательство. Пусть $\varphi-$ эндоморфизм КЛМ $Q$ и $\left\{a_{i}\right\}-$ множество ее порождающих. Покажем, что множество $\left\{\varphi a_{i}\right\}$ порождает $Q$. Действительно, согласно предположению $A(Q) \neq Q$. Тогда множество $\left\{a_{i}\right\}$ по модулю $A(Q)$ порождает $Q / A(Q)$. В [7] доказано, что в произвольной КЛМ ассоциаторная подлупа принадлежит подлупе Фраттини. Поэтому множество $\left\{\varphi a_{i}\right\}$ порождает $Q$. Обратное очевидно.

Следствие 3. Пусть $H$ - нормальная подлупа КЛМ $Q$ с условием $A(Q) \neq Q$ и пусть $H \subseteq A(Q)$. Тогда для любого автоморфизма ф фактор-лупь $Q / H$ существует автоморфизм лупь $Q$, который индуциирует $\varphi$.

Доказательство. Действительно, существует эндоморфизм КЛМ $Q$, который индуцирует автоморфизм $\varphi$ лупы $Q / H$. Так как $H \subseteq A(Q)$, этот эндоморфизм индуцирует автоморфизм на $Q / A(Q)$. По лемме 6, этот эндоморфизм является автоморфизмом.

Следствие 4. Группа автоморфизмов КЛМ $Q$ с условием $A(Q) \neq Q$ является расширением группь, состоящей из автоморфизмов, индуцирующих тождественное отображение на $Q / A(Q)$ с помощью группь автоморфизмов абелевой группь $Q / A(Q)$.

Из следствий 1, 2, 4 и леммы 4 получаем следующее утверждение.

Теорема 2. Пусть КЛМ $Q$ аппроксимируется центрально нильпотентными КЛМ. Тогда ее группа автоморфизмов является расширением нильпотентно аппроксимируемой группы $F(1)$, состоящей из всех автоморфизмов, индуциирующих тождественное отображение на $Q / A(Q)$ с помощью группь автоморфизмов абелевой группь $Q / A(Q)$. Если КЛМ $Q$ иентрально нильпотентна ступени $n$, то группа $F(1)$ нильпотентна ступени $n-1$ и показателя $3^{k}$, где $k=\max \left\{r \mid 2^{r} \leqslant n\right\}$. В частности, если КЛМ $Q$ конечно порождена, то $F(1)$ будет конечной 3-группой.

Хорошо известна теорема А. И. Мальцева [7] (см. также [8]): разрешимая группа автоморфизмов конечно порожденной абелевой группы полициклична. Однако также верно следующее утверждение. 
Предложение 2. Разрешимая группа автоморфизмов Ф конечно порожденной КЛМ $Q$ полициклична.

Доказательство. Пусть $H-$ периодическая часть КЛМ $Q$. Так как в КЛМ внутренние подстановки являются ее автоморфизмами [3], отсюда следует, что $H$ - нормальная подлупа КЛМ $Q$. Согласно [11], КЛМ $Q$ удовлетворяет условию максимальности для подлуп. Тогда $H$ конечно порождена, и из теоремы Брака-Слэби и условия максимальности легко следует, что она конечна. Рассмотрим гомоморфизм $\alpha \rightarrow \operatorname{Aut}(Q / H)$, сопоставляющий произвольному $\varphi \in \Phi$ автоморфизм $\bar{\varphi}$ фактор-лупы $Q / H$, индуцированный $\varphi$, то есть $\bar{\varphi}(a H)=\varphi(a) H$. Так как $Q / H-$ конечно порожденная абелева группа без кручения, она разлагается в прямое произведение конечного числа бесконечных циклических групп. Тогда $\operatorname{Aut}(Q / H)$ изоморфна группе целочисленных матриц с определителем \pm 1 . Согласно $[9,10]$, любая разрешимая группа целочисленных матриц полициклична. Поэтому $\Phi / \Psi$, где $\Psi=\operatorname{ker} \alpha$, есть полициклическая группа целочисленных матриц. Докажем конечность $\Psi$. Действительно, если $a_{1}, \ldots, a_{k}$ - система порождающих КЛМ $Q$, то для произвольного $\psi \in \Psi$ справедливо равенство $\psi\left(a_{i}\right)=a_{i} h_{i}, h_{i} \in H$. Ввиду конечности $H$, имеется лишь конечное число возможностей для образов порождающих $a_{i}$ при отображениях из $\Psi$. Отсюда следует конечность $\Psi$, а тогда и полицикличность $\Phi$. Предложение доказано.

Автор выражает благодарность Н. И. Санду за оказанную помощь при выполнении данной работы.

\section{Список литературы}

1. Санду Н. И., Об относительно свободных коммутативных лупах Муфанг. Алгебра и логика (1979) 18, №3, 194-205.

2. Санду Н. И., Медиально нильпотентные дистрибутивные квазигруппы и СН-квазигруппы. $\mathrm{Cu-}$ бирский матем. ж. (1987) 28, №2, 159-170.

3. Bruck R. H., Survey of binary systems. Springer, Berlin, 1958.

4. Smith J. D. H., On the nilpotence class of commutative Moufang loops. Math. Proc. Cambridge Phil. Soc. (1978) 84, №3, 387-404.

5. Malbos J.-P., Sur la classe de nilpotence des boucles commutatives de Moufang et des espaces mediaux. C. R. Acad. Sci., Paris, Sér. A (1978) 287, 691-693.

6. Beneteau L., Free commutative Moufang loops and anticommutative graded rings. J. Algebra (1980) 67, 1-35.

7. Санду Н. И., О длине нижнего центрального ряда (производного ряда) коммутативной лупы Муфанг. Математические заметки (1997) 62, №3, 475-479.

8. Санду Н. И., О центрально нильпотентных коммутативных лупах Муфанг. В сб.: Квазигруппь и лупь. Штиинца, Кишинев, 1979, с. 145-155.

9. Мальцев А. И., О некоторых классах бесконечных разрешимых групп. Математический сб. (1951) 28, №3, 567-588.

10. Каргаполов М. И., Мерзляков Ю. И., Основы теории групп. Наука, Москва, 1977.

11. Evans T., Identities and relations in commutative Moufang loops. J. Algebra (1974) 31, 508-513.

Статья поступила 11.10.2010. 